\title{
Construction reproducibility of a composite tooth model composed of an intraoral-scanned crown and a cone-beam computed tomography-scanned root
}

\author{
Seung-Weon $\operatorname{Lim}^{\mathrm{a}}$ (1) \\ Ryu-Jin Moon ${ }^{\mathrm{a}}$ \\ Min-Seok Kim ${ }^{\mathrm{b}}$ \\ Min-Hee $\mathrm{Oh}^{\mathrm{a}}$ \\ Kyung-Min Lee ${ }^{\mathrm{a}}$ \\ Hyeon-Shik Hwang ${ }^{a}$ \\ Tae-Woo Kim ${ }^{\mathrm{c}}$ \\ Seung-Hak Baek ${ }^{c}$ \\ Jin-Hyoung $\mathrm{Cho}^{\mathrm{a}}$ (1)
}

${ }^{a}$ Department of Orthodontics, School of Dentistry, Chonnam National University, Gwangju, Korea

${ }^{b}$ Department of Oral Anatomy, School of Dentistry, Chonnam National

University, Gwangju, Korea

'Department of Orthodontics, School of Dentistry, Seoul National University, Seoul, Korea
Objective: To evaluate the construction reproducibility of a composite tooth model (CTM) composed of an intraoral-scanned crown and a cone-beam computed tomography (CBCT)-scanned root. Methods: The study assessed 240 teeth (30 central incisors, 30 canines, 30 second premolars, and 30 first molars in the maxillary and mandibular arches) from 15 young adult patients whose pre-treatment intraoral scan and CBCT were available. Examiner-Reference (3 years' experience in CTM construction) and Examiners-A and Examiner-B (no experience) constructed the individual CTMs independently by performing the following steps: image acquisition and processing into a three-dimensional model, integration of intraoral-scanned crowns and CBCT-scanned teeth, and replacement of the CBCT-scanned crown with the intraoral-scanned crown. The tooth axis angle in terms of mesiodistal angulation and buccolingual inclination of the CTMs constructed by the three examiners were measured. To assess the construction reproducibility of CTMs, intraclass correlation coefficient (ICC) assessments were performed. Results: The ICC values of mesiodistal angulation and buccolingual inclination among the 3 examiners showed excellent agreement (0.950-0.992 and 0.965-0.993; 0.976-0.994 and 0.973-0.995 in the maxillary and mandibular arches, respectively). Conclusions: The CTM showed excellent construction reproducibility in mesiodistal angulation and buccolingual inclination regardless of the construction skill and experience levels of the examiners.

[Korean J Orthod 2020;50(4):229-237]

Key words: Construction reproducibility, Composite tooth model, Intraoralscanned crown, Cone-beam computed tomography-scanned root

Received November 12, 2019; Revised January 30, 2020; Accepted March 3, 2020.

Corresponding author: Jin-Hyoung Cho.

Professor and Chair, Department of Orthodontics, School of Dentistry, Chonnam National University, 33, Yongbong-ro, Buk-gu, Gwangju 61186, Korea.

Tel +82-62-530-5818 e-mail jhcho@jnu.ac.kr

Seung-Weon Lim and Ryu-Jin Moon contributed equally to this work as co-first authors.

How to cite this article: Lim SW, Moon RJ, Kim MS, Oh MH, Lee KM, Hwang HS, Kim TW, Baek SH, Cho JH. Construction reproducibility of a composite tooth model composed of an intraoral-scanned crown and a cone-beam computed tomographyscanned root. Korean J Orthod 2020;50:229-237.

(C) 2020 The Korean Association of Orthodontists.

This is an Open Access article distributed under the terms of the Creative Commons Attribution Non-Commercial License (http://creativecommons.org/licenses/by-nc/4.0) which permits unrestricted non-commercial use, distribution, and reproduction in any medium, provided the original work is properly cited. 


\section{INTRODUCTION}

Panoramic radiography has been considered to be accurate enough to evaluate the alignment of the dentition, including roots, even though it shows several shortcomings involving distortion, overlapping, and magnification. ${ }^{1,2}$ However, clinicians need to examine the root position three-dimensionally (3D) in several clinical situations such as severe skeletal malocclusion and eruption disturbances in relation to adjacent roots.

With the introduction of cone-beam computed tomography (CBCT), examination of the root position in 3D coordinates has become possible. However, because this 3D imaging technology involves more radiation exposure than panoramic radiography, repeated CBCT scans during orthodontic treatment are not recommended. ${ }^{3-5}$ In contrast, an intraoral scan can be performed without radiation exposure whenever it is required. Moreover, a crown image with a detailed occlusal surface can be obtained with a fast-capturing intraoral scanner, and its accuracy and reliability are known to be clinically acceptable..$^{6-8}$ The major drawback of this approach is the inability to represent the root.

By combining the complementary characteristics of these two 3D imaging technologies, construction of a 3D tooth model with a precise crown and root has been attempted, which we named the "composite tooth model" (CTM). ${ }^{9}$ Initially, the primary applications of CTM were for diagnosis, treatment simulation, and customized appliance fabrication in cases requiring evaluation of the crown and root simultaneously. ${ }^{10-12}$ Recently, using this CTM, monitoring root movement during and after orthodontic treatment was tried just by intraoral scanning (Figure 1). ${ }^{13,14}$ This involves the ability of the individual CTM to superimpose onto the mid- or posttreatment intraoral scan via referencing of the unaltered crown morphology as an index. Because the incorporated root tracks the crown movement, the changed root position allows visualization of the root movement (Figure 2). Therefore, once CTMs are constructed, additional CBCT scans for examining the root position at the midand post-treatment stages would not be required.

Although many trials and efforts have been performed for constructing precise 3D tooth models using various methodologies, ${ }^{10-15}$ the accuracy and reproducibility of the 3D tooth models are influenced by several steps and the construction procedures would be difficult for a novice operator. Therefore, the purposes of this study were to introduce an elaborate CTM construction procedure and to evaluate the construction reproducibility

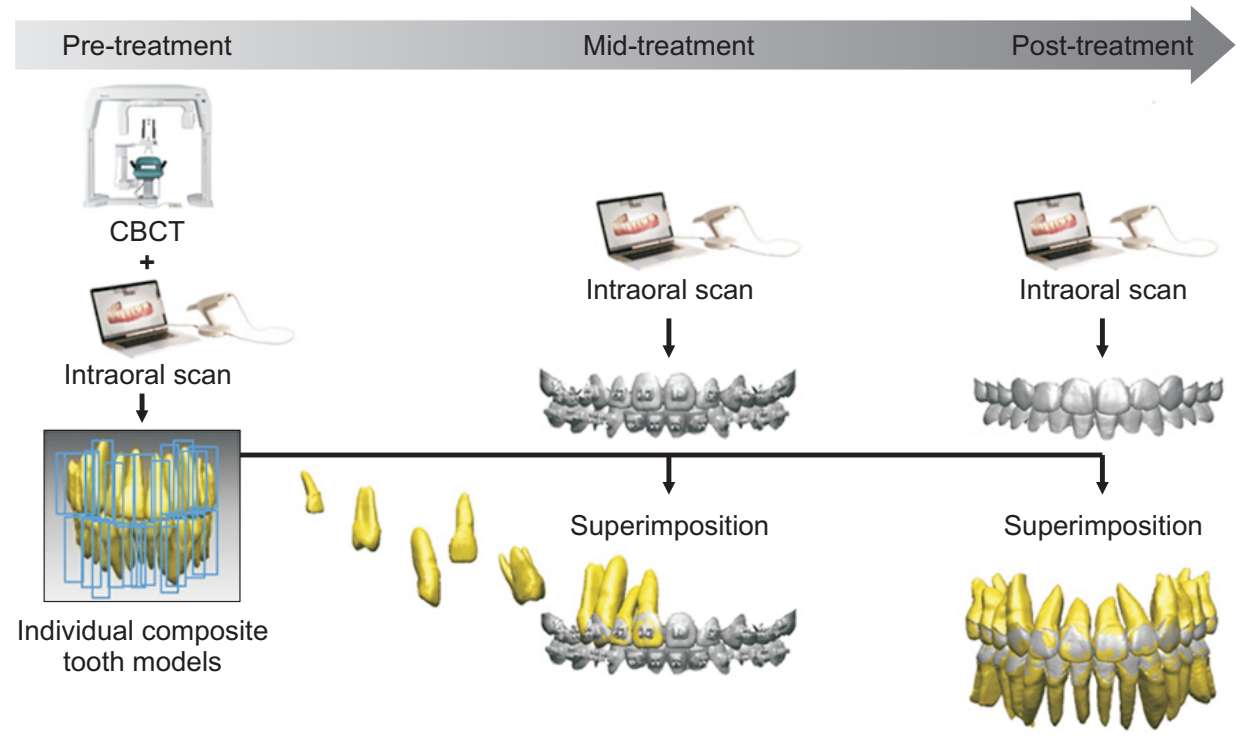

Figure 1. Clinical application of the composite tooth model (CTM). The CTMs constructed at the pre-treatment stage by combining cone-beam computed tomography (CBCT) and intraoral scan data can be used for evaluation of the root position at the mid- and post-treatment stages.
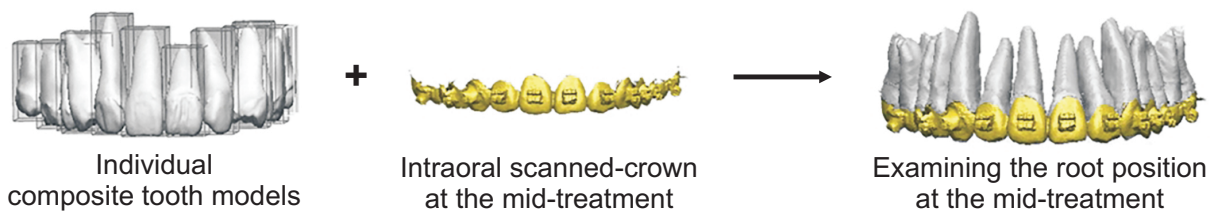

Figure 2. Evaluation of root position at the mid-treatment stage by superimposing individual composite tooth models onto the mid-treatment intraoral-scanned image using the crown as an index. 
of CTMs composed of an intraoral scanned-crown and a CBCT-scanned root.

\section{MATERIALS AND METHODS}

\section{Subjects}

The protocol for this study was reviewed and approved by the Institutional Review Board of the Chonnam National University Dental Hospital in Gwangju, Korea (No. CNUDH-2019-0003). The study included patients whose pre-treatment intraoral scans and CBCT data were available. The exclusion criteria were patients who had (1) more than two missing and/or extracted teeth, (2) more than two metal or gold crown restorations, and (3) an arch length discrepancy greater than $8 \mathrm{~mm}$ in the maxillary and mandibular arches.

To determine the sample size, a power analysis was performed based on the classification of Fleiss. ${ }^{16}$ The values of minimal and maximal intraclass correlation coefficients (ICC, $\rho 0$ and $\rho 1$ ) were set at 0.40 (fair agreement) and 0.80 (excellent agreement), respectively. With an $\alpha$ error probability of 0.05 and power of $0.8(1-\beta$ error probability), the analysis yielded a minimal sample size of 15.21 subjects for intra- and inter-examiner reproducibility assessment. Thus, 15 Korean young adult patients (8 men and 7 women; mean age, 18.9 years) were recruited. A total of 240 teeth from the 15 patients (30 central incisors $[\mathrm{Cl}], 30$ canines $[\mathrm{C}], 30$ second premolars [P2], and 30 first molars [M1] in the maxillary and mandibular arches) were tested.

\section{Examiners}

Three examiners participated in this study. One orthodontist (LSW) who had 3 years' experience in CTM construction was designated as Examiner-Reference (Examiner-Ref). Two orthodontic residents who had no experience in CTM construction were designated as Examiner-A (CIS) and Examiner-B (KHM).

To evaluate the intra-examiner reproducibility of CTM construction performed by Examiner-Ref, this examiner independently constructed the individual CTMs of all subject teeth and repeated the construction one month later. In addition, Examiner-A and Examiner-B independently constructed the individual CTMs of all subject teeth. The three sets of 240 CTMs constructed by the 3 examiners were used to evaluate the inter-examiner reproducibility in CTM construction (Figure 3).

\section{Composite tooth model construction procedure}

Step 1: Image acquisition and processing into 3D model Intraoral scanning was performed using an optical intraoral scanner $\left(\right.$ TRIOS $^{\circledR}$; 3Shape, Copenhagen, Denmark). After scanning of the maxillary and mandibular arches and removing the gingival area, the clinical crowns of maxillary and mandibular arches were converted into the stereolithography (STL) format using the OrthoAnalyzer $^{\mathrm{TM}}$ software (3Shape) (Figure 4A).

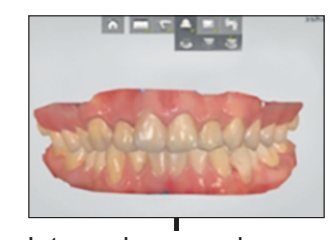

Intraoral-scanned crown by independent single operator

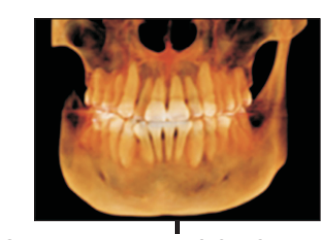

Segmentation of CBCT teeth by 3 examiners

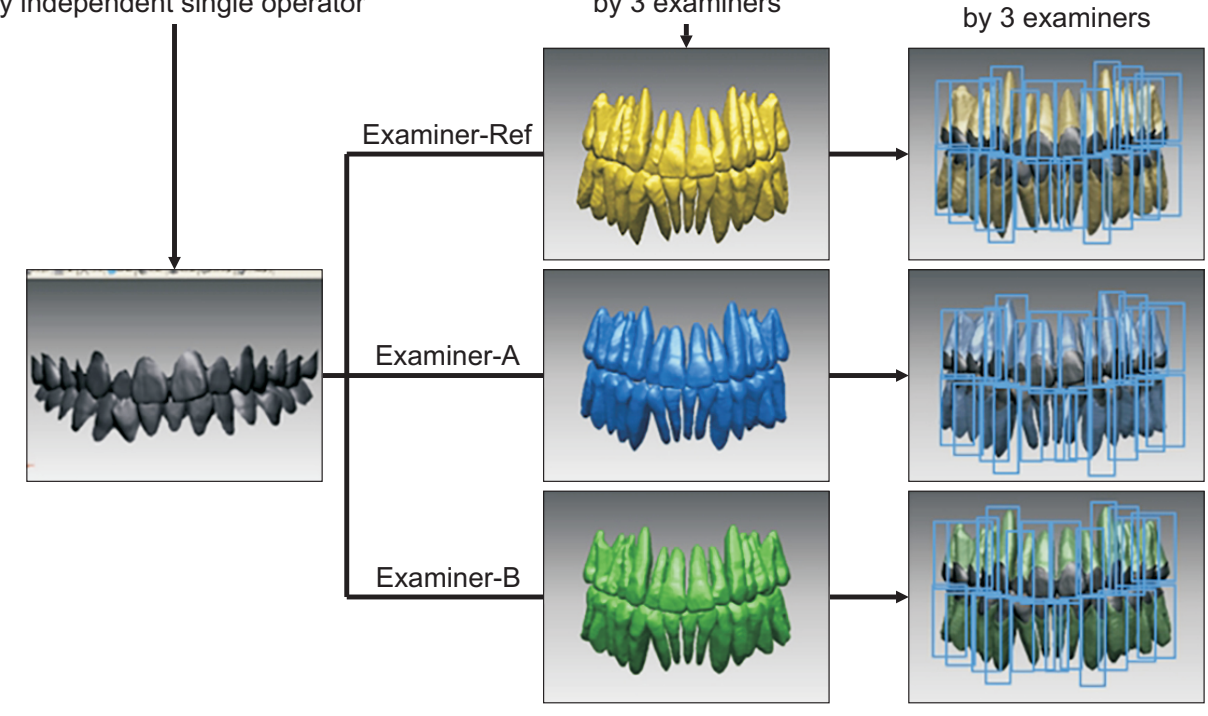

Figure 3. Study design for evaluation of inter-examiner reliability in construction of individual composite tooth models (CTMs). ExaminerReference (Examiner-Ref), Examiner- $A$, and Examiner$B$ independently constructed the individual CTMs using the intraoral-scanned crown and the cone-beam computed tomography (CBCT)-segmented teeth. 

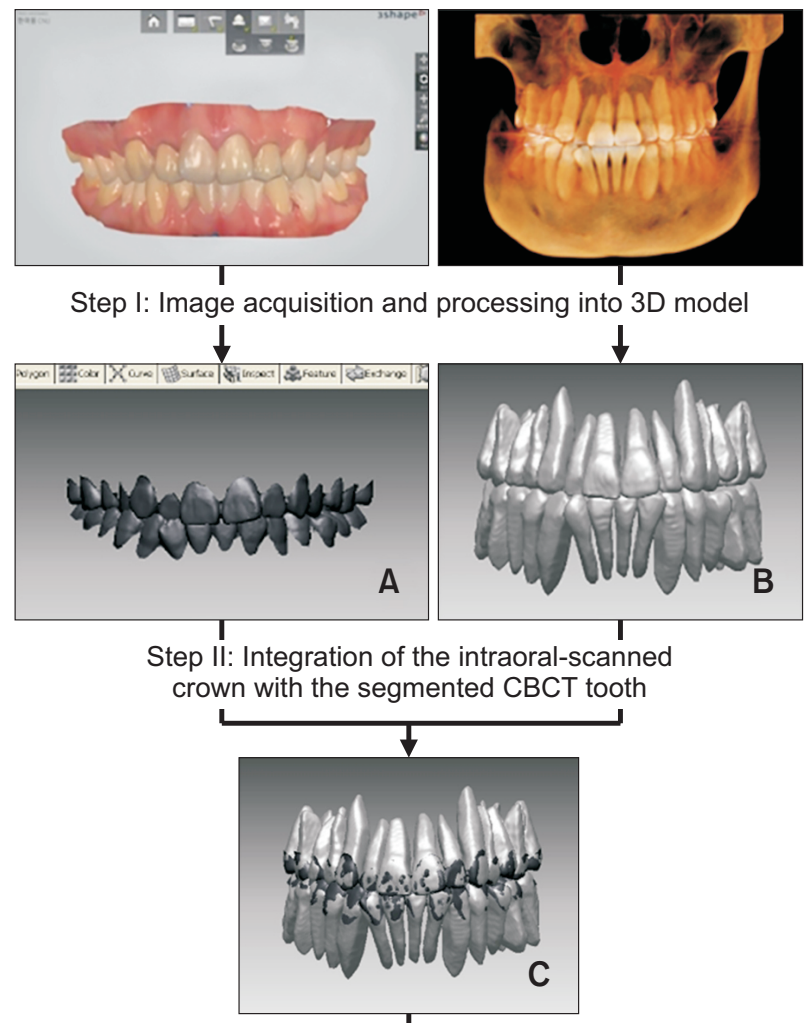

Step III: Replacement of the CBCT crown with the intraoral-scanned crown

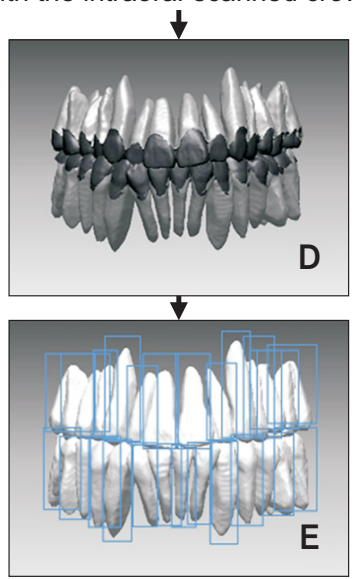

Figure 4. Construction procedure of the individual composite tooth models. A, Intraoral-scanned crown. B, Segmented cone-beam computed tomography (CBCT)scanned teeth. $\mathbf{C}$, Integration of $\mathrm{A}$ and $\mathrm{B}$ by crown registration. D, Intraoral-scanned crown with CBCT-scanned

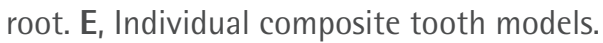

3D, Three-dimensional.

CBCT scan (Alphard Vega; Asahi Roentgen, Kyoto, Japan; $80 \mathrm{kV}$ and $5 \mathrm{~mA}$; voxel size, $0.39 \times 0.39 \times 0.39$ $\mathrm{mm}$; and field of view, $200 \times 179 \mathrm{~mm}$ ) was performed and the digital imaging and communication in medicine (DICOM) file was exported to the InVivo5 software (ver.
5.1, Anatomage, San Jose, CA, USA) for 3D volume rendering. In the "MD (medical design) studio" module, the individual tooth including the root was segmented using the "Sculpt" function and converted into the STL format (Figure 4B).

Step 11: Integration of the intraoral-scanned crowns and the segmented CBCT-scanned teeth

After the intraoral scans and CBCT 3D models from Step 1 were imported into the Rapidform ${ }^{\mathrm{TM}} 2006$ program (INUS Technology, Seoul, Korea), crown registration was performed to integrate the intraoral-scanned crown and segmented CBCT-scanned teeth. First, the initial registration was performed by selecting more than three corresponding points on each image, resulting in a rough alignment. Second, a "Fine" automatic bestfit registration was used to finalize the matches. Once the whole CBCT-scanned teeth of the maxillary arch were registered with the intraoral-scanned crowns of the maxillary arch, the mandibular arch was registered using the same method (Figure 4C).

To verify the accuracy of integration, the registration errors were evaluated using the "shell/shell deviation" function, which measures the absolute values of the 3D Euclidean distances between the surface points on the two images (Figure 5). The registration errors of the crown constructed by the 3 examiners ranged from 0.20 to $0.21 \mathrm{~mm}$ in both maxillary and mandibular arches (Table 1). Because registration errors were under 0.22 $\mathrm{mm},{ }^{9}$ we proceeded further steps below.

Step 111: Replacement of the CBCT-scanned crown with the intraoral-scanned crown

To replace the CBCT crown image with the intraoral scan, the CBCT-scanned crown was removed from the integrated image (Figure 4D). Then, the intraoralscanned crown and the CBCT-scanned root were merged using the "Merge" function of the program (Figure 4E).

\section{Measurement}

The tooth axis angle in terms of the mesiodistal angulation and buccolingual inclination of the individual CTMs was measured using two reference planes, the occlusal plane and tooth axis plane (Figure 6). The occlusal plane was defined by three points, including the midpoint of the vertical overlap between the right maxillary and mandibular $\mathrm{Cl}$ and the mesiobuccal cusp tips of the left and right maxillary $\mathrm{M} 1$. The tooth axis plane was defined by three points, including the most apical point of the root apex and two points in the crown (Table 2 and Figure 6). Then, the distal and lingual side angles between the two reference planes were computed by using the "Angle-ref. plane/ref. plane" function of Rapidform $^{\mathrm{TM}} 2006$. 


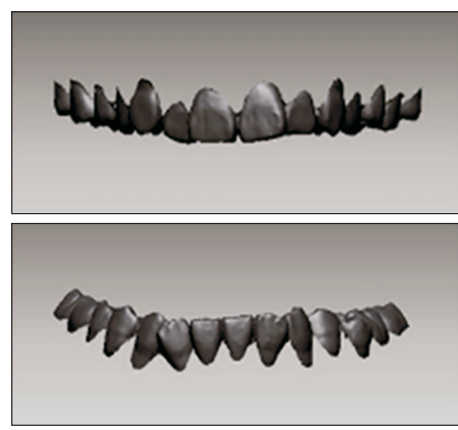

Intraoral scanned-crown

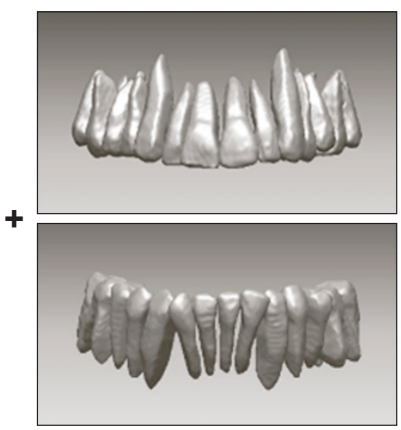

CBCT teeth

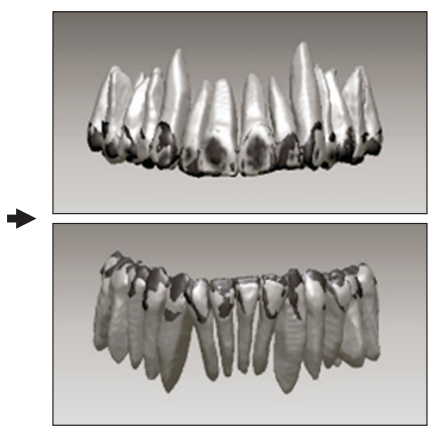

Integration

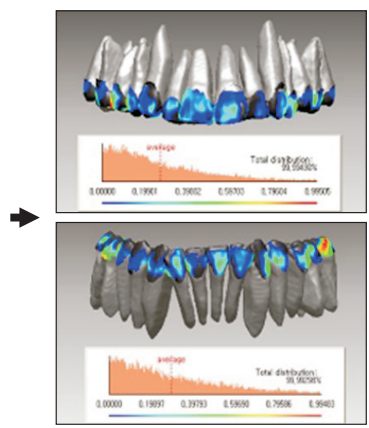

Verification

Figure 5. Verification of the integration accuracy. Registration errors were evaluated by measuring the absolute values of the three-dimensional Euclidean distances between the surface points on the two images.

$\mathrm{CBCT}$, Cone-beam computed tomography.

Table 1. Shell/shell deviation of the crowns in the maxillary and mandibular arches to verify the accuracy of integration $(n=15)$

\begin{tabular}{|c|c|c|c|c|c|c|c|}
\hline \multirow{2}{*}{$\begin{array}{c}\text { Shell/shell } \\
\text { deviation (mm) }\end{array}$} & \multicolumn{2}{|c|}{ Examiner-Ref } & \multicolumn{2}{|c|}{ Examiner-A } & \multicolumn{2}{|c|}{ Examiner-B } & \multirow{2}{*}{$\begin{array}{c}\text { ICC } \\
(95 \% \mathrm{CI})\end{array}$} \\
\hline & Mean & SD & Mean & SD & Mean & SD & \\
\hline Maxilla & 0.20 & 0.02 & 0.21 & 0.03 & 0.20 & 0.03 & 0.936 \\
\hline Mandible & 0.20 & 0.03 & 0.21 & 0.04 & 0.21 & 0.05 & 0.901 \\
\hline
\end{tabular}

Intraclass correlation coefficient (ICC) test was performed.

Examiner-Ref, Examiner-Reference; SD, standard deviation; CI, confidence interval.

\section{Statistical analysis}

Intra-examiner reproducibility of constructions performed by Examiner-Ref

Since the intra-examiner reproducibility of ExaminerRef demonstrated an excellent agreement in terms of the ICC values of the mesiodistal angulation and buccolingual inclination (range: 0.957-0.993 and 0.975-0.995, respectively; data are not shown), the CTMs constructed by Examiner-Ref were considered as the reference. Further evaluation of the inter-examiner reproducibility went on.

\section{Inter-examiner reproducibility}

Inter-examiner reproducibility of the mesiodistal angulation and buccolingual inclination was evaluated by the ICC test. Statistical analysis was performed using IBM SPSS ver. 23.0 (IBM Corp., Armonk, NY, USA).

\section{RESULTS}

\section{Inter-examiner reproducibility}

The ICC values of the mesiodistal angulation were 0.950-0.992 and 0.965-0.993 in the maxillary and mandibular arches, respectively (range of difference, $0.01-0.79^{\circ}$; Table 3). The ICC values of the buccolingual inclination were $0.976-0.994$ and 0.973-0.995 in the maxillary and mandibular arches, respectively (range of difference, $0.04-0.77^{\circ}$; Table 4).

\section{DISCUSSION}

With the application of CTMs constructed using single СВСТ at the pre-treatment stage, the 3D root position during or after treatment can be examined by intraoral scanning alone (Figures 1 and 2). Many studies have attempted to construct 3D tooth models and apply them to clinical practice. Macchi et al. ${ }^{10}$ constructed a 3Dsetup model to visualize tooth movement before and after orthodontic treatment by integrating multi-slice computed tomography and laser scans. Guo et al." applied a 3D-setup model constructed by integrating CBCT and optical scans to the indirect bonding system. Kihara et al. ${ }^{12}$ demonstrated that prediction of the root position after treatment using the 3D tooth model was reliable. Lee et al. ${ }^{13}$ used the ex-vivo typodont models to monitor tooth movement and demonstrated that root surface differences between the expected and true root position of composite teeth were $0.02 \pm 0.32 \mathrm{~mm}$ and $0.09 \pm 0.25$ $\mathrm{mm}$ in the maxillary and mandibular teeth, respectively. Subsequently, Lee et al. ${ }^{15}$ validated the accuracy and reliability of this methodology using a greater number of human subjects. Their tooth model exhibited mean differences of $1.39 \pm 1.05^{\circ}$ and $1.30 \pm 0.92^{\circ}$ in the me- 


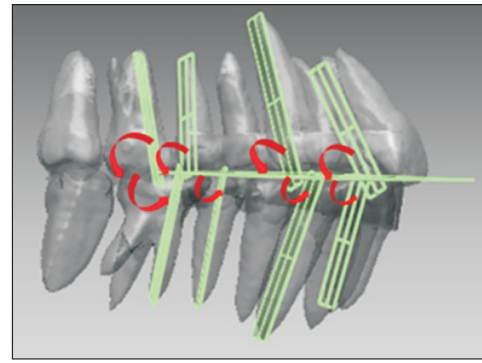

Mesiodistal angulation

(20)
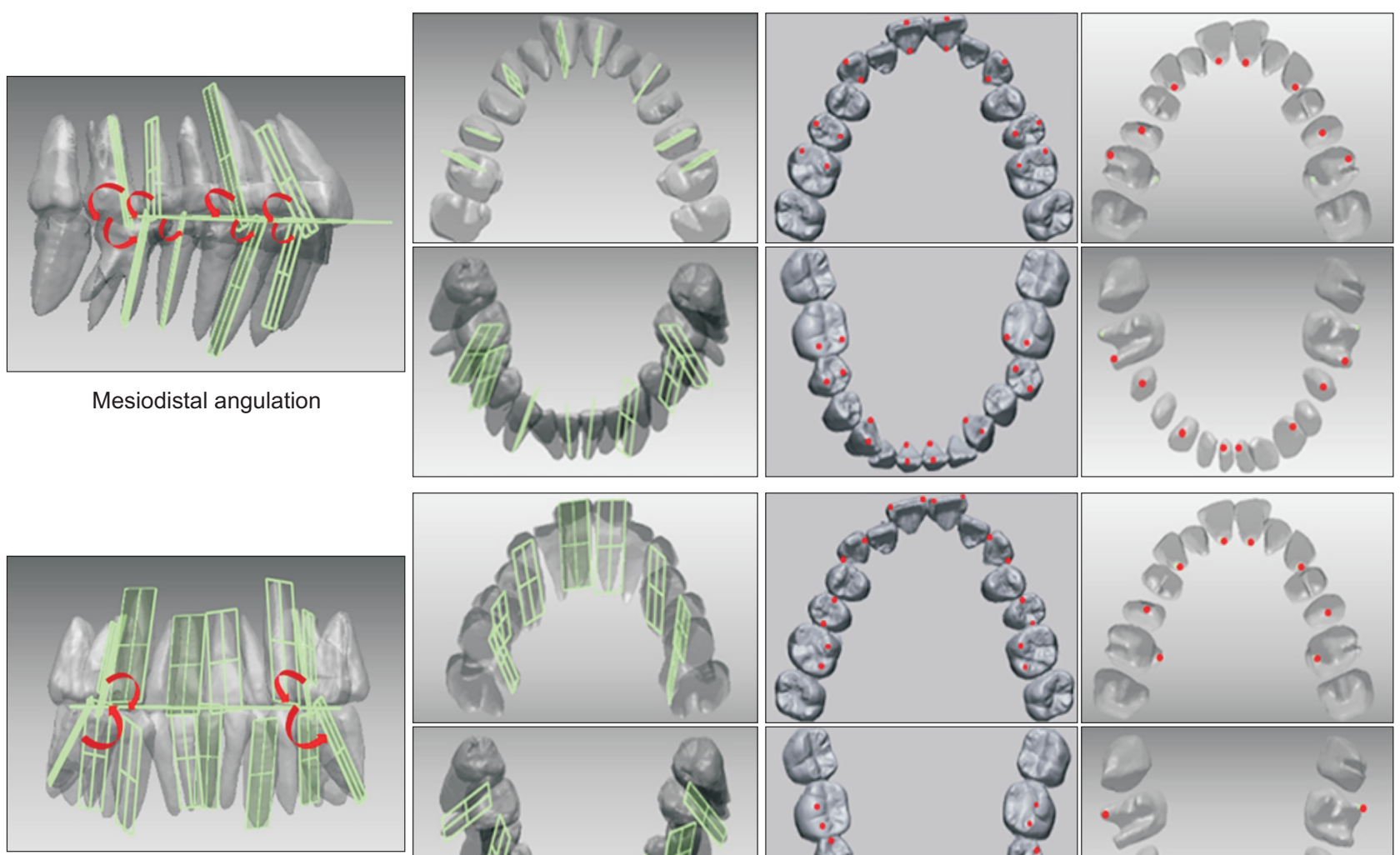

Buccolingual inclination
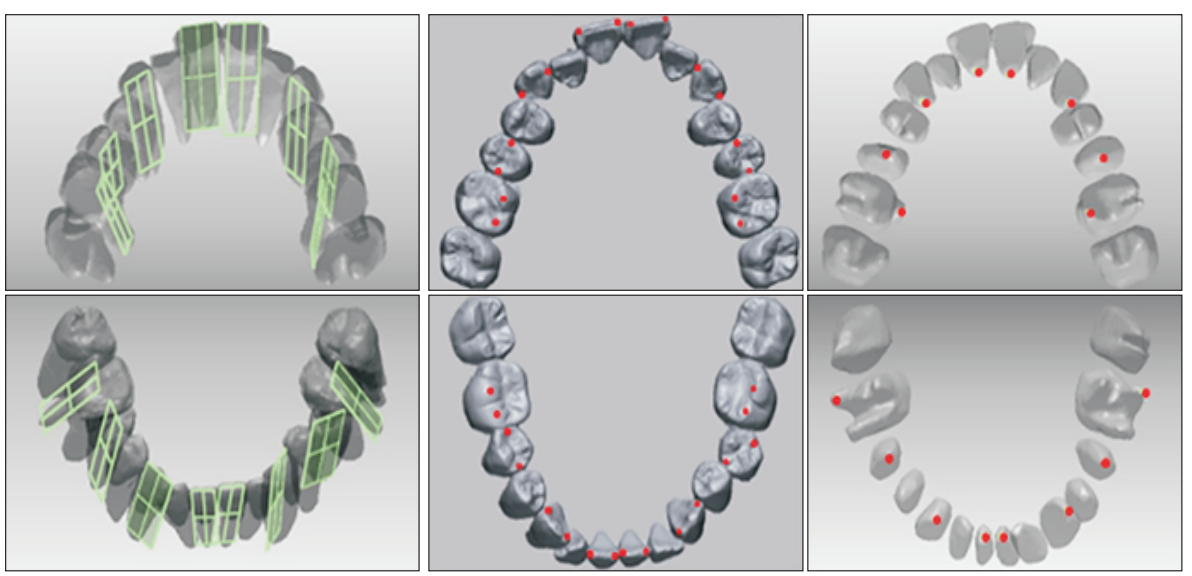

Figure 6. Measurements of tooth axis angle. The mesiodistal angulation was measured at the distal angle in conjunction with the occlusal plane and the tooth axis plane (upper row). The buccolingual inclination was measured at the lingual angle in conjunction with the occlusal plane and the tooth axis plane (lower row). The green square-shaped plane presents tooth axis planes constructed by two points in the crown and one point in the root apex.

siodistal angulation and the buccolingual inclination between the post-treatment CBCT scan and the expected root position setup, indicating high inter-operator reliability. However, $11.6 \%$ and $9.6 \%$ of teeth measured for the mesiodistal angulation and the buccolingual inclination were outside the $\pm 2.5^{\circ}$ range of clinical acceptability.

The main idea of this study was to introduce an elaborate method for constructing CTMs and to verify the CTMs constructed by this method. In this study, to evaluate the inter-examiner reproducibility in construction of CTMs, the tooth axis angles of $\mathrm{Cl}, \mathrm{C}, \mathrm{P} 2$, and M1 ( $\mathrm{n}=30 /$ tooth type) in the maxillary and mandibular arches were compared. The mesiodistal angulation and the buccolingual inclination demonstrated a high degree of inter-examiner reproducibility (ICC, 0.950-0.992 and $0.965-0.993$ in the maxillary and mandibular arches, respectively, Table 3; ICC, 0.976-0.994 and 0.973-0.995 in the maxillary and mandibular arches, respectively, Table 4). The mean differences in tooth axis angle between examiners ranged from $0.01^{\circ}$ to $0.79^{\circ}$ in the mesiodistal angulation and $0.04^{\circ}$ to $0.77^{\circ}$ in the buccolingual inclination, which can be considered to be very small under clinical conditions. These results were better than those reported by Lee et al. ${ }^{15}$ Therefore, the CTM construction methodology proposed in this study demonstrated excellent inter-examiner reproducibility regardless of the construction skill and experience level of the examiners. Although the differences between examiners were clinically acceptable (range: $0.01-0.79^{\circ}$ and $0.04-0.77^{\circ}$ in the mesiodistal angulation and buccolingual inclination, respectively), they were supposed to have originated from the tooth segmentation procedure from the CBCT. The inherent differences in root images might have resulted in inter-examiner differences (step 1), and the differences in crown images also might have resulted in differences in the integration with the intraoral scan images (step 11). As a result, steps 1 and 11 were mainly responsible for the inter-examiner differences.

In terms of the time required for CTM construction by 
Table 2. Definitions of the landmarks in the crown and root for constructing the tooth axis plane

\begin{tabular}{|c|c|c|c|}
\hline \multirow{2}{*}{$\begin{array}{l}\text { Tooth axis } \\
\text { plane }\end{array}$} & \multirow{2}{*}{ Tooth } & \multicolumn{2}{|c|}{ Definition of landmarks } \\
\hline & & Two points in the crown & One point in the root \\
\hline \multirow[t]{4}{*}{$\begin{array}{l}\text { Mesiodistal } \\
\text { angulation }\end{array}$} & Central incisor & $\begin{array}{l}\text { The most mesial and distal point of the } \\
\text { incisor edge }\end{array}$ & The most apical point of the root \\
\hline & Canine & $\begin{array}{l}\text { The most mesial and distal point of the } \\
\text { marginal ridge }\end{array}$ & The most apical point of the root \\
\hline & Second premolar & $\begin{array}{l}\text { The most mesial and distal point in the } \\
\text { line of occlusion }\end{array}$ & $\begin{array}{l}\text { Max: The most apical point of the palatal } \\
\text { root }\end{array}$ \\
\hline & First molar & $\begin{array}{l}\text { Max: The mesiopalatal and distopalatal } \\
\text { cusp tips } \\
\text { Man: The mesiobuccal and distobuccal } \\
\text { cusp tips }\end{array}$ & $\begin{array}{l}\text { Max: The most apical point of the palatal } \\
\text { root } \\
\text { Man: The most apical point of the distal } \\
\text { or distobuccal root }\end{array}$ \\
\hline \multirow[t]{4}{*}{$\begin{array}{l}\text { Buccolingual } \\
\text { inclination }\end{array}$} & Central incisor & $\begin{array}{l}\text { The midpoint of the incisor edge and the } \\
\text { central pit of lingual surface }\end{array}$ & The most apical point of the root \\
\hline & Canine & The cusp tip and cingulum & The most apical point of the root \\
\hline & Second premolar & The buccal and lingual cusp tips & $\begin{array}{l}\text { Max: The most apical point of the buccal } \\
\text { root }\end{array}$ \\
\hline & First molar & $\begin{array}{l}\text { Max: The mesiobuccal and mesiopalatal } \\
\text { cusp tips } \\
\text { Man: The mesiobuccal and mesiolingual } \\
\text { cusp tips }\end{array}$ & $\begin{array}{l}\text { Max: The most apical point of the } \\
\text { mesiobuccal root } \\
\text { Man: The most apical point of the mesial } \\
\text { or mesiobuccal root }\end{array}$ \\
\hline
\end{tabular}

Max, Maxilla; Man, mandible.

Table 3. Mesiodistal angulation $\left({ }^{\circ}\right.$ ) of composite tooth models constructed by the 3 examiners $(\mathrm{n}=30 /$ tooth type)

\begin{tabular}{|c|c|c|c|c|c|c|c|c|}
\hline \multirow{2}{*}{\multicolumn{2}{|c|}{ Tooth }} & \multicolumn{2}{|c|}{ Examiner-Ref } & \multicolumn{2}{|c|}{ Examiner-A } & \multicolumn{2}{|c|}{ Examiner-B } & \multirow{2}{*}{$\begin{array}{c}\text { ICC } \\
(95 \% \mathrm{CI})\end{array}$} \\
\hline & & Mean & SD & Mean & SD & Mean & SD & \\
\hline \multirow{4}{*}{ Maxilla } & Central incisor & 88.28 & 4.83 & 88.04 & 5.46 & 88.51 & 5.38 & 0.950 \\
\hline & Canine & 79.49 & 7.45 & 79.90 & 7.07 & 79.80 & 7.55 & 0.992 \\
\hline & 2nd premolar & 83.75 & 6.30 & 83.89 & 6.65 & 83.88 & 6.13 & 0.992 \\
\hline & 1st molar & 83.02 & 7.55 & 83.23 & 7.41 & 82.81 & 7.99 & 0.980 \\
\hline \multirow[t]{4}{*}{ Mandible } & Central incisor & 91.39 & 5.13 & 91.83 & 5.33 & 91.04 & 5.59 & 0.965 \\
\hline & Canine & 84.57 & 7.01 & 84.35 & 7.18 & 84.58 & 6.76 & 0.989 \\
\hline & 2nd premolar & 79.76 & 6.24 & 79.45 & 6.27 & 79.84 & 5.81 & 0.993 \\
\hline & 1st molar & 79.53 & 5.18 & 79.38 & 4.96 & 79.60 & 4.86 & 0.989 \\
\hline
\end{tabular}

Intraclass correlation coefficient (ICC) test was performed.

Examiner-Ref, Examiner-Reference; SD, standard deviation; CI, confidence interval.

the novice operators, Examiners-A and Examiner-B, it took about 14-15 hours for the total of 28 teeth in one patient. On the other hand, for Examiner-Ref, who had had three years' experience in CTM construction, it took about 10-11 hours. Repeated construction improved the proficiency in construction, thereby reducing the operating time by the learning effect. ${ }^{17}$ Unfortunately, we could not plot the learning curve. The most timeconsuming procedure was tooth segmentation from CBCT scans, which occupied about two-thirds of the total time. Because fully automated tooth segmentation has not been verified yet, ${ }^{18}$ human intervention was unavoidable. Although the smaller voxel size results in an image with finer detail and higher contrast, ${ }^{19}$ artifacts such as beam hardening, truncation, and scattered radiation can affect the contrast and definition of the bone and root. ${ }^{20,21}$ Additionally, streak artifacts from brackets or metal restorations make it difficult to segment the crown from CBCT images. ${ }^{21}$

The application of the CTM would be clinically valuable because no additional radiation exposure is required after the initial CBCT. However, CTM construc- 
Table 4. Buccolingual inclination $\left({ }^{\circ}\right)$ of composite tooth models constructed by the 3 examiners $(n=30 /$ tooth type)

\begin{tabular}{|c|c|c|c|c|c|c|c|c|}
\hline & \multirow{2}{*}{ Tooth } & \multicolumn{2}{|c|}{ Examiner-Ref } & \multicolumn{2}{|c|}{ Examiner-A } & \multicolumn{2}{|c|}{ Examiner-B } & \multirow{2}{*}{$\begin{array}{c}\text { ICC } \\
(95 \% \mathrm{CI})\end{array}$} \\
\hline & & Mean & SD & Mean & SD & Mean & SD & \\
\hline \multirow[t]{4}{*}{ Maxilla } & Central incisor & 55.44 & 4.22 & 54.81 & 4.20 & 55.01 & 4.02 & 0.992 \\
\hline & Canine & 70.51 & 9.21 & 70.55 & 9.30 & 70.11 & 9.23 & 0.994 \\
\hline & 2nd premolar & 88.02 & 8.27 & 88.22 & 8.60 & 87.93 & 8.41 & 0.976 \\
\hline & 1st molar & 80.12 & 6.20 & 79.96 & 6.50 & 79.43 & 6.71 & 0.987 \\
\hline \multirow[t]{4}{*}{ Mandible } & Central incisor & 71.04 & 8.50 & 71.50 & 7.71 & 70.91 & 8.86 & 0.993 \\
\hline & Canine & 78.55 & 8.03 & 78.58 & 8.05 & 78.92 & 8.48 & 0.995 \\
\hline & 2nd premolar & 92.86 & 7.85 & 92.81 & 7.84 & 92.99 & 7.27 & 0.973 \\
\hline & 1st molar & 95.53 & 9.24 & 94.76 & 9.05 & 95.13 & 9.19 & 0.990 \\
\hline
\end{tabular}

Intraclass correlation coefficient (ICC) test was performed.

Examiner-Ref, Examiner-Reference; SD, standard deviation; CI, confidence interval.

tion should be restricted to patients who underwent the initial CBCT for justified reasons, such as severe skeletal discrepancy, craniofacial deformity, and ectopic erupting teeth. Thus, the initial CBCT scans can be utilized not only for diagnosis, but also for examining changes in root position. Routine initial CBCT for orthodontic diagnosis cannot be justified, as reported in the "guidelines on the CВCT for dental and maxillofacial radiology' by SEDENTEXCT. ${ }^{22}$ All of the included subjects in this study were patients who were likely to be candidates for orthognathic surgery.

On the other hand, there are several shortcomings in СTM. To assess any pathologic changes such as external root resorption and bony change, additional radiography would be required. Moreover, numerous technical aspects need improvement before CTMs can be reliably used under clinical conditions. Most importantly, the labor-intensive and time-consuming nature of this approach remains to be addresses. In this regard, adoption of auto-segmentation technology and development of all-in-one software for CTM may help increase operation efficiency.

This study was a preliminary study for validation of CTM in root position estimation at the mid- or posttreatment stage. Investigation of the applications of CTMs at mid-treatment intraoral scans would be performed in the near future, as a next step.

\section{CONCLUSION}

CTM constructed by the proposed methodology showed an excellent construction reproducibility in tooth axis angle regardless of the construction skill and experience levels of the examiners.

\section{CONFLICTS OF INTEREST}

No potential conflict of interest relevant to this article was reported.

\section{ACKNOWLEDGEMENTS}

This research was supported by a grant from the Korea Health Technology REtD Project through the Korea Health Industry Development Institute (KHIDI), funded by the Ministry of Health \& Welfare, Korea (grant number: $\mathrm{HI} 18 \mathrm{C} 1638$ ) and a grant from the Chonnam National University Hospital Biomedical Research Institute (CRI 18044-21).

We would like to extend our gratitude to Dr. HyunHee Choo for the intraoral scanning procedure, as well as Dr. Hyun-Min Kim and Dr. In-Sun Choi for the construction of the composite tooth model; all three were residents in the Department of Orthodontics, School of Dentistry, Chonnam National University Dental Hospital (CNUDH), Gwangju, Korea.

We thank Dr. Il-Sik Cho (Seoulbarun orthodontic clinic, Pohang, Korea) for the comments on the statistical analysis.

\section{REFERENCES}

1. Garcia-Figueroa MA, Raboud DW, Lam EW, Heo G, Major PW. Effect of buccolingual root angulation on the mesiodistal angulation shown on panoramic radiographs. Am J Orthod Dentofacial Orthop 2008;134:93-9.

2. Mckee IW, Glover KE, Williamson PC, Lam EW, Heo G, Major PW. The effect of vertical and horizontal head positioning in panoramic radiography on mesiodistal tooth angulations. Angle Orthod 2001;71:442-51.

3. Song IT, Cho JH, Chae JM, Chang NY. Evaluation of 
mesiodistal tooth axis using a CBCT-generated panoramic view. Korean J Orthod 2011;41:255-67.

4. Lascala CA, Panella J, Marques MM. Analysis of the accuracy of linear measurements obtained by cone beam computed tomography (CBCT-NewTom). Dentomaxillofac Radiol 2004;33:291-4.

5. Tong H, Enciso R, Van Elslande D, Major PW, Sameshima GT. A new method to measure mesiodistal angulation and faciolingual inclination of each whole tooth with volumetric cone-beam computed tomography images. Am J Orthod Dentofacial Orthop 2012;142:133-43.

6. Ender A, Mehl A. Full arch scans: conventional versus digital impressions--an in-vitro study. Int J Comput Dent 2011;14:11-21.

7. Hayashi K, Sachdeva AU, Saitoh S, Lee SP, Kubota T, Mizoguchi 1. Assessment of the accuracy and reliability of new 3-dimensional scanning devices. Am J Orthod Dentofacial Orthop 2013;144:619-25.

8. Sun L, Lee JS, Choo HH, Hwang HS, Lee KM. Reproducibility of an intraoral scanner: a comparison between in-vivo and ex-vivo scans. Am J Orthod Dentofacial Orthop 2018;154:305-10.

9. Lim SW, Hwang HS, Cho IS, Baek SH, Cho JH. Registration accuracy between intraoral-scanned and cone-beam computed tomography-scanned crowns in various registration methods. Am J Orthod Dentofacial Orthop 2020;157:348-56.

10. Macchi A, Carrafiello G, Cacciafesta V, Norcini A. Three-dimensional digital modeling and setup. Am J Orthod Dentofacial Orthop 2006;129:605-10.

11. Guo H, Zhou J, Bai Y, Li S. A three-dimensional setup model with dental roots. J Clin Orthod 2011; 45:209-16; quiz 235-6.

12. Kihara T, Tanimoto K, Michida M, Yoshimi Y, Nagasaki T, Murayama T, et al. Construction of orthodontic setup models on a computer. Am J Orthod Dentofacial Orthop 2012;141:806-13.

13. Lee RJ, Pham J, Choy M, Weissheimer A, Dougherty HL Jr, Sameshima GT, et al. Monitoring of typodont root movement via crown superimposition of single cone-beam computed tomography and consecutive intraoral scans. Am J Orthod Dentofacial Orthop
2014;145:399-409.

14. Lee RJ, Weissheimer A, Pham J, Go L, de Menezes LM, Redmond WR, et al. Three-dimensional monitoring of root movement during orthodontic treatment. Am J Orthod Dentofacial Orthop 2015;147:132-42.

15. Lee RJ, Pi S, Park J, Devgon D, Nelson G, Hatcher D, et al. Accuracy and reliability of the expected root position setup methodology to evaluate root position during orthodontic treatment. Am J Orthod Dentofacial Orthop 2018;154:583-95.

16. Walter SD, Eliasziw M, Donner A. Sample size and optimal designs for reliability studies. Stat Med 1998;17:101-10.

17. Schunk DH. Learning theories: an educational perspective. 6th ed. Boston: Pearson Education; 2012. p. 38-40.

18. Wang C, Liu Y, Wang S, Wang Y, Zhao Y. Evaluation of in vivo digital root reconstruction based on anatomical characteristics of the periodontal ligament using cone beam computed tomography. Sci Rep 2018;8:269.

19. Ye N, Long H, Xue J, Wang S, Yang X, Lai W. Integration accuracy of laser-scanned dental models into maxillofacial cone beam computed tomography images of different voxel sizes with different segmentation threshold settings. Oral Surg Oral Med Oral Pathol Oral Radiol 2014;117:780-6.

20. Marmulla R, Wörtche R, Mühling J, Hassfeld S. Geometric accuracy of the NewTom 9000 Cone Beam CT. Dentomaxillofac Radiol 2005;34:28-31.

21. Sang $\mathrm{YH}, \mathrm{Hu} \mathrm{HC}$, Lu SH, Wu YW, Li WR, Tang ZH. Accuracy assessment of three-dimensional surface reconstructions of in vivo teeth from cone-beam computed tomography. Chin Med J (Engl) 2016; 129:1464-70.

22. European Commission. Cone Beam CT for dental and maxillofacial radiology: evidence-based guidelines [Internet]. Luxembourg: SEDENTEXCT; 2012 Mar [cited 2020 Jan 30]. Available from: http:// www.sedentexct.eu/files/radiation_protection_172. pdf. 